\title{
Focusing of a tabletop soft-x-ray laser beam and laser ablation
}

\author{
B. R. Benware, A. Ozols, ${ }^{*}$ and J. J. Rocca \\ Department of Electrical and Computer Engineering, Colorado State University, Fort Collins, Colorado 80523
}

\author{
I. A. Artioukov, V. V. Kondratenko, and A. V. Vinogradov \\ P. N. Lebedev Physical Institute, Moscow, 117924 Russia
}

Received August 12, 1999

\begin{abstract}
We focused the beam of a high-repetition-rate capillary-discharge tabletop laser operating at a wavelength of $46.9 \mathrm{~nm}$, using a spherical $\mathrm{Si} / \mathrm{Sc}$ multilayer mirror. The energy densities significantly exceeded the thresholds for the ablation of metals. Single-shot laser ablation patterns were used in combination with ray-tracing computations to characterize the focused beam. The radiation intensity within the 2- $\mu$ m-diameter central region of the focal spot was estimated to be $\approx 10^{11} \mathrm{~W} / \mathrm{cm}^{2}$, with a corresponding energy density of $\sim 100 \mathrm{~J} / \mathrm{cm}^{2}$. ( $) 1999$ Optical Society of America

OCIS codes: $\quad 140.7240,340.7470,340.7440$.
\end{abstract}

Soft-x-ray lasers have been shown to have sufficient intensity to produce high-resolution images in a single shot. ${ }^{1,2}$ Recent advances in table-top sources of soft$\mathrm{x}$-ray coherent radiation have created the possibility of generating short-wavelength radiation pulses of unprecedentedly high peak intensities at high repetition rates. $^{3} \quad$ Focusing of these intense soft-x-ray beams will open new applications, including nonlinear optics at ultrashort wavelengths, nanomachining, and generation of plasmas with soft-x-ray photons. High-order harmonic pulses with wavelengths near $55 \mathrm{~nm}$ and subpicosecond duration were recently focused to reach an estimated intensity of $1 \times 10^{11} \mathrm{~W} / \mathrm{cm}^{2} .{ }^{4}$ Focusing of tabletop soft-x-ray lasers, which are now capable of producing millijoule-level pulses,${ }^{5}$ should also yield very high intensities while allowing for much higher energy density. Recently, Ohchi et al. focused nanojoule pulses from the $15.47-\mathrm{nm}$ line of Li-like $\mathrm{Al}$ into a submicrometer spot to obtain an intensity of $\approx 2 \times 10^{7} \mathrm{~W} / \mathrm{cm}^{2}{ }^{6} \quad$ Preliminary results of an attempt to focus laser pulses of much higher energy from a collisional soft-x-ray laser pumped by a large optical laser have been also reported. ${ }^{7}$ In this Letter we report the generation and characterization of a focused soft-x-ray laser beam with intensity and energy density that significantly exceed the threshold for the ablation of metals. We obtained these results by focusing 0.13-mJ laser pulses of 1.2-ns FWHM duration produced by a tabletop capillary-discharge Ne-like Ar laser $(\lambda=46.9 \mathrm{~nm})$ with a spherical $\mathrm{Si} / \mathrm{Sc}$ multilayer mirror.

The experimental setup is shown in Fig. 1. The laser pulses were generated at a repetition rate of $1 \mathrm{~Hz}$ by amplification in an 18.2-cm-long Ar capillary plasma that was excited with a fast current pulse. $^{8}$ The far-field laser beam profile had an annular shape and a peak-to-peak divergence of $\sim 4.6 \mathrm{mrad}^{8}{ }^{8}$ The laser intensity distribution near the exit of the amplifier was verified to also be annular; the unfocused laser beam ablated a ring pattern with a peak-to-peak diameter of $\approx 340 \mu \mathrm{m}$ onto a piece of acrylic placed $1.1 \mathrm{~cm}$ from the end of the capillary. The annular intensity distribution is caused by refraction of the rays in the amplifier owing to radial density gradients. ${ }^{9,10}$ The laser beam was focused with a spherical $(R=10 \mathrm{~cm}) \mathrm{Si} / \mathrm{Sc}$ multilayer-coated mirror positioned in a vacuum chamber at $256.5 \mathrm{~cm}$ from the exit of the capillary-discharge amplifier. The multilayer coating was deposited by magnetron sputtering $^{11}$ on a 2.5-cm-diameter superpolished borosilicate substrate with a rms surface roughness of $0.1 \mathrm{~nm}$. We measured the normal-incidence reflectivity of these multilayer coatings to be $\approx 43 \%$ at $46.9 \mathrm{~nm}$. The mirror was positioned at normal incidence with the purpose of minimizing aberrations, and the reflected beam was focused onto the narrowest side of a thin metal strip ( $1.6 \mathrm{~mm}$ thick, $\approx 1 \mathrm{~cm}$ wide). For the measurements reported here we employed a brass target, but similar results were obtained with stainless steel. Note that in this setup the target also intercepts part of the incoming beam. However, this is not a problem because the target blocks only a small fraction of the incoming beam, which at that location

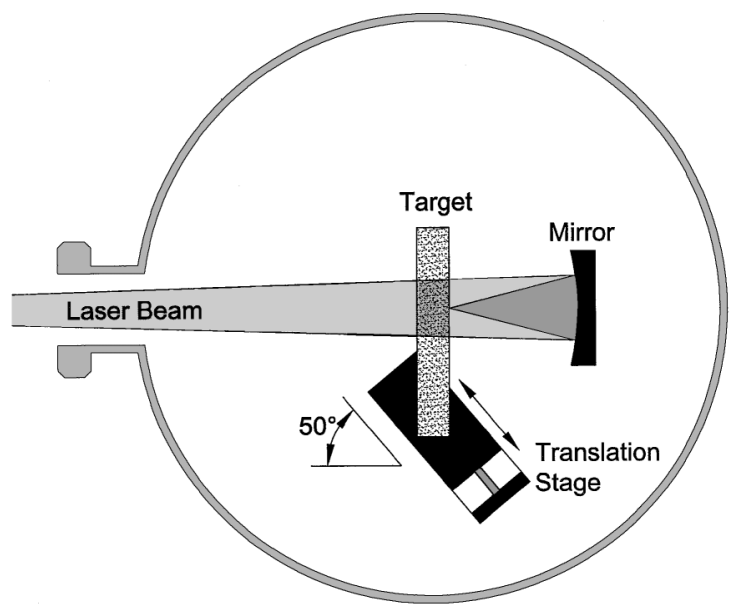

Fig. 1. Experimental setup used to focus and characterize the soft-x-ray laser beam.

(C) 1999 Optical Society of America 
has a diameter of $\sim 12 \mathrm{~mm}$. The focused laser beam was observed to readily ablate metals within several hundred micrometers of the focus, creating a visible plasma plume. The imprints on the metal surface give useful two-dimensional information on the focused laser beam intensity distribution, in spite of the dependence of the size of the molten region on the heat conductivity, melting point of the sample material, and duration of the laser pulse. ${ }^{12}$ To monitor the focused beam intensity distribution at different planes along the propagation axis we mounted the target on a translation stage driven by a computer-controlled stepper motor. The axis of motion of the translation stage was positioned at an angle of $50^{\circ}$ with respect to the optical axis. We obtained a series of imprints of the beam profile along the optical axis by continuously moving the translation stage while repetitively firing the laser at $1 \mathrm{~Hz}$.

Figure 2 is a scanning electron microscope (SEM) photograph showing the progression of ablation patterns obtained as the target was moved away from the mirror toward the focus. The figure shows the convergence of the beam within an axial region of $\sim 450 \mu \mathrm{m}$ near the focus. The sampling distance between any two contiguous shots is $22.2 \mu \mathrm{m}$ along the optical axis (note that this axial distance between patterns is smaller than it appears in the SEM photographs because the sample is moved at an angle with respect to the optical axis). Each ablation pattern is the result of a single laser shot. Figure 3 shows part of the same sequence with larger magnification. At a distance of a few hundred micrometers from the focal region the ablated patterns have the shape of thin annular disks. The patterns have good azimuthal symmetry, except for a small discontinuity where the incoming beam was blocked by the target. As the focal region is approached, the thickness of the ablated rings is observed to increase, and an ablated spot develops at the center. The depth of these annular grooves was measured with a visible laser interferometer to be $\approx 2 \mu \mathrm{m}$. Finally, very near the focus the patterns evolve into a single spot with a deep hole on axis. The smallest ablated spot has a diameter of $\sim 17 \mu \mathrm{m}$ and contains a deep hole of $\approx 3-\mu \mathrm{m}$ diameter on axis. Not shown in Fig. 3 are the ablation patterns that we obtained by positioning the target on the other side of the focus at increasing distances from the mirror, which rapidly become diffused and more difficult to distinguish.

We conducted ray-tracing computations to analyze the results and obtain an estimate of the power density that was achieved. The beam was approximated assuming a point source located $263 \mathrm{~cm}$ from the mirror, with an angular distribution tailored to match closely the measured laser beam intensity in the near and far fields. We obtained similar results by simulating the source with a $300-\mu \mathrm{m}$-diameter disk. We computed the trajectory of $1.3 \times 10^{4}$ rays to simulate the beam propagation. Figure 4 shows the computed cross section of the beam intensity distributions in the focal region. For comparison with the experiment the measured boundaries of the ablated regions are represented by filled circles in Fig. 4. All the major features of the observed ablation profiles of Figs. 2 and 3 are well described by the ray-tracing computations. The computations

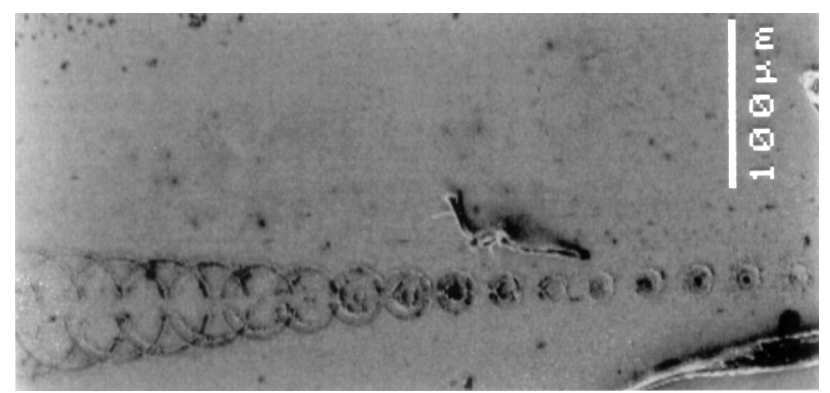

Fig. 2. SEM image of soft-x-ray laser ablation patterns on the surface of a brass sample, illustrating the convergence of the focused beam.
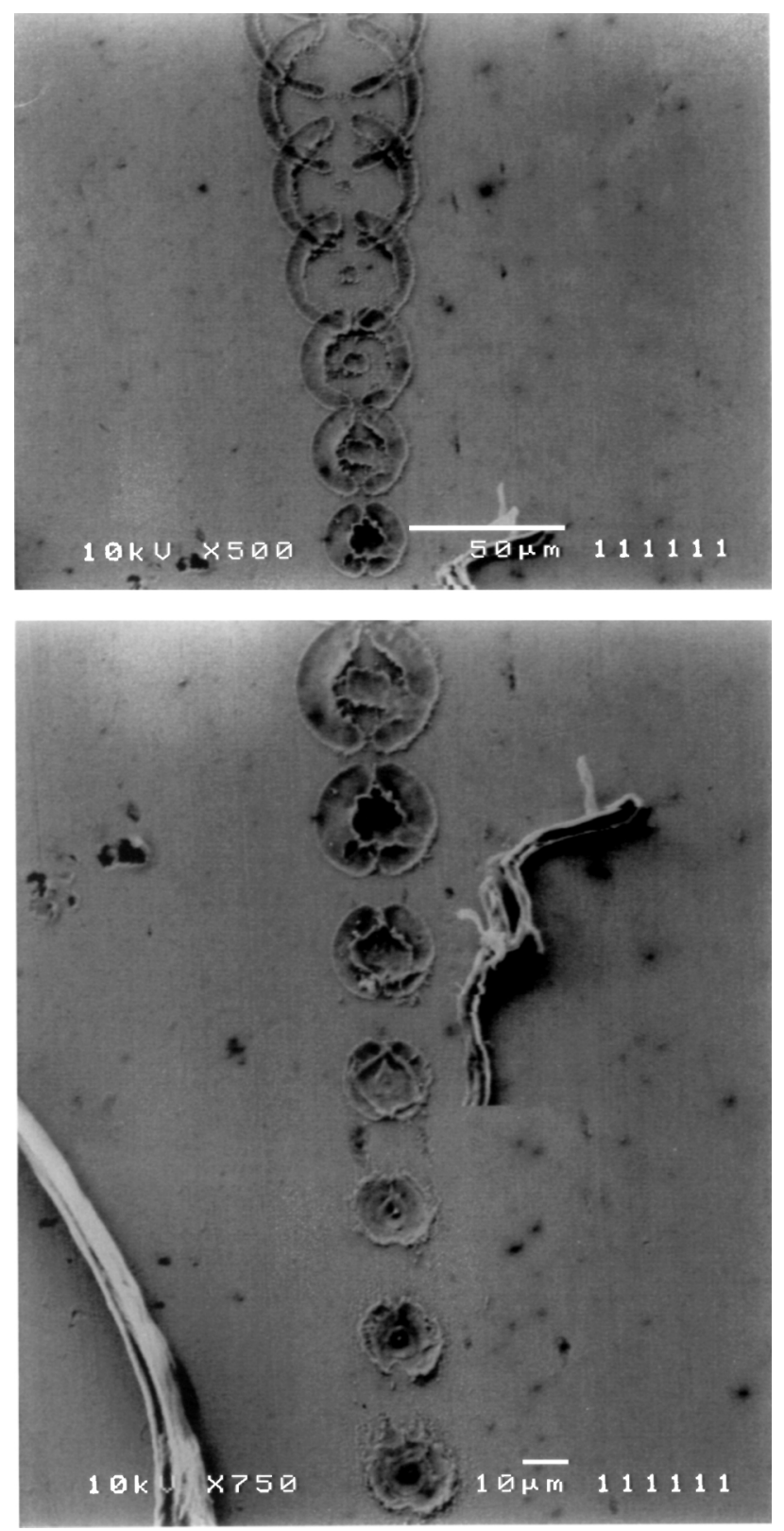

Fig. 3. SEM images of the ablation patterns in the focal region. 


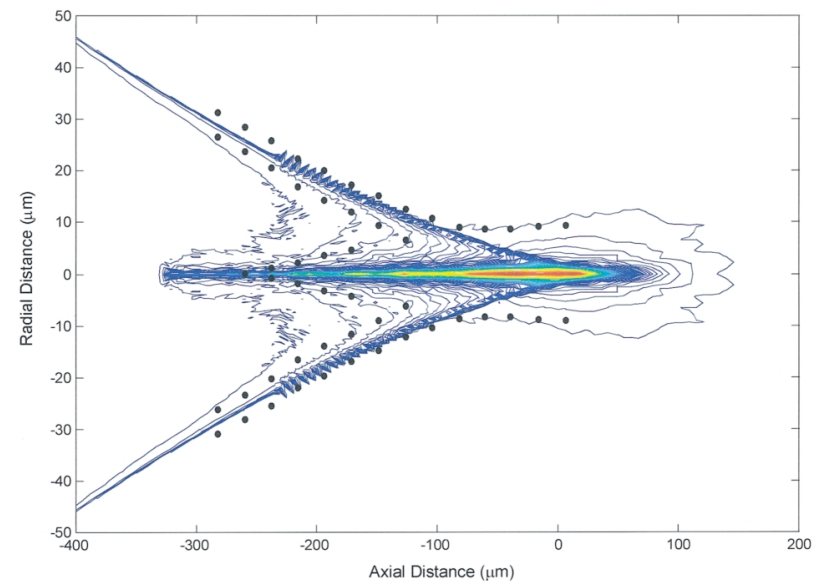

Fig. 4. Computed cross section of the beam in the focal region. The filled circles are the measured boundaries of the patterns ablated on a brass sample.

show that a few hundred micrometers from the focal region the highest concentration of rays defines thin annular disks. Also in accordance with the experiment, a high intensity peak is observed at the axis as the focal region is approached. Both features are the result of the spherical aberration that causes the rays to converge and cross at these locations. Therefore the observed annular ablation patterns are not a direct consequence of the annular profile of the laser beam. The spherical aberration would also cause similar rings for any beam profile with a sufficient intensity in the periphery (such as a flat intensity distribution). Likewise, the spherical aberration causes the central peak, which begins to develop when the outermost rays converge on axis. Near the so-called plane of minimum confusion the intensity distribution is computed to be dominated by the sharp central peak, which causes the deep holes observed at the center of the ablation patterns shown near the bottom of Fig. 3. The average intensity within a $2-\mu \mathrm{m}$-diameter central region is estimated from the computed fraction of rays that intersect this region to be $\approx 1 \times 10^{11} \mathrm{~W} / \mathrm{cm}^{2}$. The corresponding energy density within this central region is $\approx 100 \mathrm{~J} / \mathrm{cm}^{2}$. This analysis also confirms that the minimum spot size is determined predominantly by the spherical aberration and not by the partial spatial coherence of the laser beam. ${ }^{13}$

In summary, a spherical multilayer mirror was used to focus high-energy laser pulses $(0.13 \mathrm{~mJ})$ generated by a 46.9-nm tabletop capillary-discharge amplifier, yielding what we believe to be the first clear demonstration of material ablation with a coherent softx-ray beam. The peak intensities are estimated to be $\approx 1 \times 10^{11} \mathrm{~W} / \mathrm{cm}^{2}$, dominated by the spherical aberration. Considering that Ne-like Ar laser pulses with energy of nearly $1 \mathrm{~mJ}$ were recently generated at a $4-\mathrm{Hz}$ repetition rate ${ }^{5}$ intensities of the order of $1 \mathrm{TW} \mathrm{cm}^{-2}$ should be readily obtained with the same focusing setup, opening new applications for tabletop soft-x-ray lasers. The use of specially shaped focusing optics should allow for significantly higher intensities.

We acknowledge the support of U.S. Department of Energy grant DE-FG03-98DP00208. The laser was developed with the support of National Science Foundation grant DMR-9512282. We also acknowledge the support of the U.S. Civilian Research and Development Foundation grant RPI-240 and the Colorado Advanced Technology Institute and are indebted to J. L. A. Chilla, Yu. S. Kasyanov, and A. P. Kanavin for important discussions. J. J. Rocca's e-mail address is rocca@ engr.colostate.edu.

*Permanent address, Facultad de Ingenieria, Universidad de Buenos Aires, Buenos Aires, Argentina.

\section{References}

1. D. S. Di Cicco, D. Kim, R. Rosser, and S. Suckewer, Opt. Lett. 17, 157 (1992)

2. L. B. Da Silva, J. A. Trebes, R. Balhorn, S. Mrowka, E. Anderson, D. T. Atwood, T. W. Barbee, Jr., J. Brase, M. Corzett, J. Gray, J. A. Koch, C. Lee, D. Kern, R. A. London, B. J. McGowan, D. L. Matthews, and G. Stone, Science 258, 269 (1992).

3. J. J. Rocca and L. B. Da Silva, eds., Proc. SPIE 3776 (1999).

4. L. Le Déroff, P. Salières, and B. Carré, Opt. Lett. 23, 1544 (1998).

5. C. D. Macchietto, B. R. Benware, and J. J. Rocca, Opt. Lett. 24, 1115 (1999).

6. T. Ohchi, N. Yamaguchi, C. Fujikawa, and T. Hara, in $X$-Ray Lasers 1998, Y. Kato, H. Takuma, and H. Daido, eds., Vol. 159 of IOP Conference Proceedings (Institute of Physics, London, 1998), p. 683.

7. P. Zeitourn, S. Sebban, K. Murai, H. Tang, Ph. Troussel, F. Albert, H. Daido, G. de Lacheze-Murel, P. Dhez, A. Carillon, G. Cauchon, Y. Gu, G. Huang, S. Hubert, G. Jamelot, Y. Kato, A. Mirone, N. Sakaya, S. Wang, D. Hambach, and B. Niemann, in X-Ray Lasers 1998, Y. Kato, H. Takuma, and H. Daido, eds., Vol. 159 of IOP Conference Proceedings (Institute of Physics, London, 1998), p. 677.

8. B. R. Benware, C. D. Macchietto, C. H. Moreno, and J. J. Rocca, Phys. Rev. Lett. 81, 5804 (1998).

9. C. H. Moreno, M. C. Marconi, V. N. Shlyaptsev, B. R. Benware, C. D. Macchietto, J. L. A. Chilla, J. J. Rocca, and A. L. Osterheld, Phys. Rev. A 58, 1509 (1998).

10. J. L. A. Chilla and J. J. Rocca, J. Opt. Soc. Am. B 13, 2841 (1996).

11. Yu. A. Uspenskii, V. E. Lebashov, A. V. Vinogradov, A. I. Fedorenko, V. V. Kondratenko, Yu. P. Pershin, E. N. Zubalev, and V. Yu. Fedotov, Opt. Lett. 23, 771 (1998).

12. J. C. Miller and R. F. Haglund, Laser Ablation and Desorption (Academic, San Diego, Calif., 1998).

13. M. C. Marconi, J. L. A. Chilla, C. H. Moreno, B. R. Benware, and J. J. Rocca, Phys. Rev. Lett. 79, 2799 (1997). 